\title{
HISTORIA DE LA EDUCACIÓN MATEMÁTICA. PRESENTACIÓN
}

\section{History of Mathematics Education. Presentation}

\author{
María Teresa GonzÁlez Astudillo \\ Universidad de Salamanca \\ Correo-e: maite@usal.es \\ Wagner Rodrigues VAlente \\ Universidad Federal de São Paulo \\ Correo-e: wagner.valente@unifesp.br
}

\begin{abstract}
Codo indica que la Historia de la Matemática como campo disciplinar mantiene una larga tradición en todos los países. En España, por ejemplo, hay que destacar esta línea de investigación en la Universidad de Zaragoza, que se mantiene desde hace décadas. Pero a medida que crece el movimiento de profesionalización de la docencia, y de la docencia de la Matemática, se ha producido una ramificación de ese campo dando la posibilidad de la creación de la Historia de la Educación Matemática. Se sabe que, a partir de un campo disciplinar, surgen nuevas disciplinas, nuevos desmembramientos del campo inicial, ramificando el rango de posibilidades de construcción de nuevos campos de investigación e incluso profesionales:
\end{abstract}

El proceso de disciplinarización no se completa con la generalización del sistema de disciplinas, pero prosigue incansable bajo múltiples formas. Las disciplinas se constituyen frecuentemente unas en relación con otras, en un movimiento incesante de reconfiguración [...] (Hofstetter y Schneuwly, 2017, p. 25).

La Historia de la Educación Matemática (HEM) es un ejemplo de esa ramificación y reconfiguración. A partir de la inclusión de las posibilidades didácticopedagógicas de la Historia de la Matemática en la enseñanza, se produce una revalorización e incremento de los estudios sobre el papel de la Historia en la enseñanza de la matemática.

Los trabajos que tratan sobre historia de la educación matemática se han multiplicado en el ámbito internacional. Cabría citar como ejemplos la consolidación de eventos como el Congreso Iberoamericano de Historia de la Educación Matemática (CIHEM), que celebró su quinta edición en 2019 en Bogotá, Colombia. 
En las ediciones anteriores había reunido investigadores en esta área en Portugal, México, Brasil y España. También debe citarse la realización de la Conference on the History of Mathematics Education (ICHME), cuya sexta edición se realizó también en el año 2019 en Marsella, Francia, y que reúne investigadores del ámbito internacional que trabajan esta temática. Además, hay que mencionar que también en el ámbito internacional el ICME (International Congress on Mathematics Education), que se celebra cada cuatro años, mantiene un Topic Group desde la edición de 2016 en Hamburgo (Alemania) dedicado a la Historia de la Educación Matemática.

Más allá de estos los eventos científicos internacionales, se han empezado a publicar revistas periódicas específicas en el área como HISTEMAT - Revista de História da Educação Matemática, publicación de la Sociedade Brasileira de História da Matemática.

En España, en el seno de la Sociedad Española de Investigación en Educación Matemática (SEIEM), hay un grupo de investigación sobre Historia de la Matemática y de la Educación Matemática que se creó en el año 2003 y que aglutina investigadores de diversas universidades españolas como Salamanca, Murcia, Granada, Córdoba, Valencia y Zaragoza. Estos investigadores han realizado y dirigido numerosas tesis doctorales en este ámbito incluso desde antes de que se creara el grupo y tienen un papel muy activo en el seno de esta Sociedad y de los simposios que se organizan. Prueba de ello es que en el séptimo simposio celebrado en Granada en el año 2003 y en el VII y en el decimotercero celebrado en Valladolid en el año 2019 se celebraron sendos seminarios temáticos dedicados a la Historia de la Educación Matemática.

Este monográfico reúne contribuciones de autores iberoamericanos que sistematizaron resultados de investigación en el campo de la Historia de la Educación Matemática y que buscan un diálogo aún más amplio con historiadores de la educación, por medio de Historia de la Educación. Revista Interuniversitaria. Son estudios que abordan temas variados de la enseñanza de la matemática, en diversos niveles educativos, con la movilización de una amplia gama de documentos que han servido como fuentes de investigación.

El primer artículo, escrito por Wagner Valente, de la Universidade Federal de São Paulo, Brasil, analiza la enseñanza de la matemática en los primeros años escolares durante la segunda mitad del siglo xIx. El estudio trata el tema de las fracciones, tal como aparecen en los libros didácticos utilizados en la época. El autor muestra cómo ocurren los cambios en la aritmética escolar debido a la adopción del sistema métrico decimal. Sigue a ese estudio, considerando el nivel secundario y universitario, un análisis de libros didácticos españoles editados en el siglo XIX, realizado por Isabel M. ${ }^{a}$ Sánchez Sierra, IES Francisco Salinas, y María Teresa González Astudillo, de la Universidad de Salamanca, que investigan las concepciones de los autores de dichos libros en relación con la Geometría Analítica. Por medio del análisis de los libros, las autoras muestran las diferentes nomenclaturas utilizadas para tratar la Geometría Analítica, además de dos concepciones diferentes sobre esta temática. 
El tercer artículo del monográfico, elaborado por Iran Abreu Mendes, de la Universidade Federal do Pará, Brasil, aborda el trabajo de Vilhena Alvez, personaje que ejerció su oficio en la región norte de Brasil, divulgando actividades matemáticas en los primeros años del siglo xx. El estudio muestra cómo Alvez articula diferentes saberes matemáticos considerando una enseñanza intuitiva.

Los cambios en la formación del profesor de matemática son abordados por medio del estudio de la tele-escuela portuguesa en la década de 1960, tema de análisis de Mária Cristina Almeida, de la Unidade de Investigação, Educação e Desenvolvimento, Portugal, y José Manuel Matos, de la Universidade Federal de Juiza de Fora, Minas Gerais, Brasil. El estudio trata de las apropiaciones del llamado Movimiento de las Matemáticas Modernas divulgado por aulas que fueron televisadas.

Dolores Carrillo Gallego, José Francisco Castejón Mochón y Pilar Olivares Carrillo, de la Universidad de Murcia, analizan, por medio de los Cuadernos Rubio, un best-seller didáctico, los problemas aritméticos considerando las diferentes ediciones de ese material.

Siguen a esos estudios el trabajo de Vanesa Pacheco Moros y Fredy Enrique González, de la Universidad de Carabobo, Venezuela, dedicado a la historia de la constitución de lo que es considerado el primer programa de investigación en Educación Matemática en el ámbito latinoamericano.

Finaliza el monográfico Alejandra Deriard, del Instituto de Formación Docente Bernardo Houssay, Argentina, que, por medio de una vasta documentación, analiza las transformaciones de la enseñanza de la matemática en Buenos Aires, considerando las propuestas de autores franceses en la segunda mitad del siglo xx, reunidos bajo la denominación de Didáctica Matemática francesa.

Como se puede observar, hay un gran alcance y variedad en los textos que componen este monográfico dedicado a la Historia de la Educación Matemática. Todos ellos, sin embargo, se articulan en torno a las transformaciones ocurridas en los saberes matemáticos. Y ese movimiento de cambios en la matemática considera la enseñanza, la formación de profesores y la investigación vinculada a la educación matemática.

Esperamos que esos estudios, representativos de diversos países, puedan contribuir a nuevas investigaciones y a la ampliación del diálogo, sobre todo con los historiadores de la educación.

\section{Bibliografía}

Hofstetter, Rita y Schneuwly, Bernard: «Disciplinarização e disciplinação: as ciências e as didáticas das disciplinas sob análise», en Hofstetter, Rita y Valente, Wagner R. (orgs.): Saberes em (trans)formação: tema central da formação de professores. São Paulo: Editora Livraria da Física, 2017, pp. 21-54.

Mendes, I. A.: Cartografias da produção em História da Matemática no Brasil: um estudo centrado nas dissertações e teses defendidas entre 1990-2010. Relatório de Pesquisa (Bolsa produtividade CNPq) - Universidade Federal do Rio Grande do Norte, Natal, 2014. 
